\title{
Chromosomal evolution and phylogenetic analyses in Tayassu pecari and Pecari tajacu (Tayassuidae): tales from constitutive heterochromatin
}

\author{
F. ADEGA ${ }^{1}$, R. CHAVES ${ }^{1}$ and H. GUEDES-PINTO ${ }^{1,2}$ \\ ${ }^{1}$ Laboratório Associado Institute for Biotechnology and Bioengineering (IBB), Centre of Genetics and Biotechnology, \\ University of Trás-os-Montes and Alto Douro (CGB-UTAD), P-5001-801 Vila Real, Portugal \\ ${ }^{2}$ School of Natural Resources, The University of Arizona, Tucson, AZ 85721, USA
}

\begin{abstract}
The mammalian family Tayassuidae (peccaries) is confined to the New World and comprises three recognized extant species, white-lipped (Tayassu pecari), collared (Pecari tajacu) and chacoan (Catagonus wagneri) peccaries, which exhibit distinct morphological and chromosomal features. The phylogenetic relationships among the tayassuids are unclear and have instigated debate over the palaeontological, cytogenetic and molecular aspects. Constitutive heterochromatin analysis can be used in understanding the phylogenetic relationships between related species. Here we describe, for the first time, the constitutive heterochromatin (C-positive heterochromatin) of two tayassuid species, Tayassu pecari and Pecari tajacu. We demonstrate that in situ restriction endonuclease digestion with sequential C-banding could be a complementary tool in the study of constitutive heterochromatin heterogeneity in chromosomes of the Tayassuidae. Our characterization of peccary chromosomes suggests that the Pecari tajacu autosomal karyotype is more primitive and has accumulated great diversity in its constitutive heterochromatin. This idea is supported by several other studies that analysed nuclear and mitochondrial sequences of the living peccary species. Finally, the tayassuid X chromosome primitive form seems to be the one of Tayassu pecari.
\end{abstract}

\section{Introduction}

Constitutive heterochromatin $(\mathrm{CH})$ represents a substantial proportion of eukaryotic genomes. The ubiquity and persis- tence of heterochromatin in these genomes suggest that this distinctive genomic component has an adaptive value, be- sides being considered a genomic compartment carrying out important functions with an unconventional structural and functional basis (Dimitri et al. 2004). Among other repetitive sequences, this genomic fraction comprises satellite DNAs that usually reside in the centromeric regions of chromo- somes (Chaves et al. 2000). Identification of $\mathrm{CH}$ and its size differences is made feasible by the simple but extremely useful C-banding technique. But other analytical methods are indispensable when the

For correspondence, e-mail: filadega@utad.pt; rchaves@utad.pt; hgp@utad.pt. molecular nature of $\mathrm{CH}$ is the central concern. Among these, use of restriction endonucleases (which cleave DNA at specific sequences) and use of base-specific fluorochromes (which delimit chromosome regions rich in different types of DNA bases) are techniques that have proven to be very useful in improving understanding of the mechanisms involved in the evolution of $\mathrm{CH}$ in different genomes (reviewed in Gosálvez et al. 1997; Pieczarka et al. 1998), In addition, restriction enzyme treatment followed by C-banding is useful in demonstrating C-heterochromatin heterogeneity (Rocco et al. 2002; Schmid et al. 2002; Chaves et al. 2004; Adega et al. 2005).

The number, morphology and G-banding pattern (e.g. Pirtle 1967; Hufty et al. 1973; Giannoni et al. 1982a,b; Benirschke and Kumamoto 1989; Zijlstra et al. 1997) of the peccaries Pecari tajacu (collared peccary) and Tayassu pecari (white-lipped peccary) are well known, and even

Keywords. constitutive heterochromatin; C-positive heterochromatin; in situ restriction endonuclease digestion; karyotype evolution; phylogeny; Pecari tajacu; Tayassu pecari. 
molecular cytogenetic studies, such as rRNA gene assignments in T. pecari (Bosma et al. 2004) and comparative chromosome painting with pig (Bosma et al. 2004; Adega et al. 2006) and sheep paint probes in both species (Adega et al. 2006; and unpublished data) are available, but their $\mathrm{CH}$ was only analysed by classical C-banding techniques (Pirtle 1967; Benirschke and Kumamoto 1989).

In this work, we describe in detail heterochromatin heterogeneity of the two peccary species $P$. tajacu and T. pecari using a panel of eight restriction endonucleases (RE) followed by the classical C-banding technique. The in situ REbanding revealed in the chromosomes was demonstrated to be suitable for studying chromosome organization. RE digestion with sequential C-banding allowed detailed characterization of $\mathrm{CH}$ in terms of its localization, detection of different $\mathrm{CH}$ subclasses, and molecular composition.

Since these two species are related, and the syntenic regions as well as the chromosomal evolutionary rearrangements (relative to another suiform genome, the pig) are known (Bosma et al. 2004; Adega et al. 2006), it was possible to track the C-positive heterochromatin along the chromosomes of the two species, and hence to delineate a hypothesis on the evolutionary remodelling of heterochromatin in these species. Further, this approach is a contribution to the understanding of the chromosomal evolutionary process and phylogenetic relationships within this family.

\section{Materials and methods}

\section{Chromosome preparation and karyotype description}

The material analysed consists of chromosome preparations of the tayassuid species $T$. pecari (three individuals) and P. tajacu (four individuals) and were made from short-term lymphocyte cultures of whole blood samples using standard protocols (Chaves et al. 2002).

The karyotype of $T$. pecari (individuals from South America) has 26 chromosomes, with the two largest chromosome pairs being submetacentric, nine pairs metacentric/submetacentric, the smallest autosomal pair acrocentric, the sex chromosome $\mathrm{X}$ a medium acrocentric, and the $\mathrm{Y}$ a small acrocentric.

The P. tajacu karyotype (individuals from Arizona, USA, North America) is characterized by a diploid chromosome number of 30, with eight metacentric/submetacentric autosomal pairs, six autosomal chromosome pairs acrocentric, the sex chromosome $\mathrm{X}$ a medium submetacentric, and the $\mathrm{Y}$ a small acrocentric. The nomenclature used for the chromosomes of T. pecari is that of Bosma et al. (2004), and for $P$. tajacu that of Adega et al. (2006).

\section{GTD-banding}

Air-dried slides were aged at $65^{\circ} \mathrm{C}$ for $5 \mathrm{~h}$, or overnight, and then were submitted to standard G-banding procedures with trypsin (Verma and Babu 1995). As the chromosome slides proceeded sequentially to C-banding, they were not stained at this stage. Instead, the slides were first fixed with paraformaldehyde (Chaves et al. 2002). Briefly, dry slides were placed in a $1 \times$ PBS solution $(2 \times 5 \mathrm{~min})$ before fixation in $4 \%$ paraformaldehyde $/ 1 \times$ PBS at room temperature. After a 10-min fixation, slides were dehydrated for 2 min each in 70, 90 and 100\% chilled ethanol, and air dried. Slides were stained with DAPI for better contrast (Chaves et al. 2002). Inversion of the DAPI colour in Adobe Photoshop software revealed the chromosomes G-banding pattern (GTD-banding, G-bands by trypsin with DAPI).

\section{In situ RE digestion}

Air-dried slides were aged at $65^{\circ} \mathrm{C}$ for $6 \mathrm{~h}$ and then were submitted to in situ RE digestion. The eight REs used (AluI, ApaI, BamHI, DraI, HaeIII, MboI, PstI and RsaI) were diluted in buffers as indicated by the manufacturer (Invitrogen, Life Technologies, Stratchclyde, UK), and final concentrations of $30 \mathrm{U}$ per $100 \mu \mathrm{l}$ were obtained. One hundred $\mu \mathrm{l}$ of each of these solutions was placed on slides and covered with coverslips. The slides were incubated in a moist chamber for $16 \mathrm{~h}$ at $37^{\circ} \mathrm{C}$. Control slides were given the same treatment as described above but incubated only with buffer. The slides were then washed in distilled water and air dried. As the chromosome slides proceeded to sequential C-banding techniques, they were fixed with paraformaldehyde (Chaves et al. 2002), as described above for GTD-banding. Finally, the slides were stained with DAPI (inversion of the DAPI colour revealed the RE-banding). The residual bands obtained after $\mathrm{RE}$ digestion were suitable for chromosome identification.

\section{CBP-banding sequential to G-bands or RE-bands}

The C-banding technique was performed sequentially to G- bands, or to RE-banding, and after destaining the slides. CBP-banding (C-bands by barium hydroxide using propidium iodide) was done using the standard procedure of Sumner (1972) but with propidium iodide as a counterstain. The use of either Giemsa or propidium iodide counterstain produces results similar to C-band identification. However, there are some technical limitations that must be considered. For example, the intensity of the bands in RE treatments seems related to the type of counterstain used (e.g. Giemsa or fluorochromes) (Gosálvez et al. 1991). Therefore, it is not advisable to compare results obtained by using different counterstains. Briefly, the slides were submitted to routine C-banding with classical treatment times reduced, approximately, to half: hydrochloric acid $(0.1 \mathrm{M}) 20 \mathrm{~min}$, barium hydroxide (5\% solution) $7 \mathrm{~min}$ and $2 \times$ saline sodium citrate ( $2 \times \mathrm{SSC}$ : $0.3 \mathrm{~mol} / \mathrm{NaCl}, 0.03 \mathrm{~mol} / 1$ sodium citrate) at $60^{\circ} \mathrm{C}$ for only $40 \mathrm{~min}$. The slides were counterstained with 1.5 $\mu \mathrm{g} / \mathrm{ml}$ propidium iodide. The images in figures 1 and 2 are representative of at least 35 metaphases observed (for each RE used), in no less than five experiments carried out at different times. 


\section{Chromosome observation}

Chromosomes were observed with a Zeiss Axioplan 2 imag- ing microscope coupled to an Axiocam digital camera with AxioVision software version 2.0.5 (Zeiss, Gö ttingen, Ger- many). Digitized photos were prepared for printing in Adobe Photoshop version 5.0; contrast and colour optimization were the functions used, and they affected the whole of the image equally.

\section{Results and discussion}

We used eight different REs in chromosome preparations of $T$. pecari and $P$. tajacu. The action of all eight REs and RE+C-banding on peccary chromosomes are presented in figures 1 and 2 . In the left column of figures 1 and 2, we present control G-banding and C-banding of the chromo- somes, which are the comparative controls for the analysis of the eight RE actions. The G-banding technique was used for the identification of chromosomes in the control experi- ment of C-banding (without previous in situ RE digestion).

The residual RE bands (figures 1 and 2, left column in each RE presented) are mainly G-like and suitable for chromosome identification. One would expect as many banding patterns as there are REs available, since the DNA cleavage should be different for each RE. However, most of the banding patterns obtained by treatment with different REs overlap. Some REs (e.g. ApaI, PstI, RsaI) produced a higher banding contrast. The banding patterns demonstrated for each RE are reproducible and can be used in sequential experiment procedures without loss of chromosome morphology.

The C-positive heterochromatin (figures 1 and 2, right chromosome in each column, control C-band and $\mathrm{RE}+\mathrm{C}$ band presented) is mainly found at the centromeres of most chromosomes, although some C-bands are observed at interstitial locations. In the individuals analysed, some heterochromatin polymorphisms of minor significance were detected, as observed for pig chromosomes (Adega et al. 2005), but these chromosome polymorphisms did not interfere with the heterochromatin patterns presented and discussed in the present work.

\section{Constitutive heterochromatin (C-positive heterochromatin) characterization in T. pecari}

In figure 1 , the $\mathrm{C}$-positive heterochromatin (C-bands) is presented for all $T$. pecari chromosomes. All the autosomal chromosomes exhibit centromeric C-bands, except chromosome 4 that shows no detectable C-positive heterochromatin at this location. At the centromere location of chromosomes 5 and 6 , it is possible to observe large Cpositive heterochro- matin blocks. Chromosomes 1, 2 and 4 show interstitial $\mathrm{C}$ - positive heterochromatin. Telomeric C-bands can be seen on chromosomes 1, 2, 3 and 4. The $\mathrm{X}$ and $\mathrm{Y}$ chromosomes also present C-positive heterochromatin regions. The acrocentric $\mathrm{X}$ chromosome exhibits centromeric, interstitial and telomeric C-blocks.
The Yq-arm appears heterochromatic with no detectable Cpositive heterochromatin at the centromeric region.

When we applied REs and sequential C-banding to the chromosomes, it was possible to verify the C-band heterogeneity (figure 1), similarly to what has been observed in other species (Chaves et al. 2004; Adega et al. 2005).

The arrowheads in figure 1 indicate the $\mathrm{C}$-bands revealed only after the RE treatment. These bands were not disclosed by classical C-banding procedures. These C-positive bands, only disclosed by previous RE treatments, could be explained by the fact that RE digestion might increase the capacity of the stain (in the present work, propidium iodide) to bind to a specific chromosome region (Gosálvez et al. 1997; Nieddu et al. 1999; Chaves et al. 2004). Most of these bands are interstitial. Chromosome 1 is the autosome revealing the highest number of C-positive heterochromatin bands. Also interesting is the clear intra-C-band revealed on the $\mathrm{X}$ chromosome after $\mathrm{MboI}+\mathrm{C}$-banding. It seems that the $\mathrm{X}$ centromeric $\mathrm{C}$ band (figure 1) with this $\mathrm{RE}+\mathrm{C}$ treatment split into two evident bands.

In T. pecari chromosomes (cf. figure 1), it was possible to identify at least three major classes of $\mathrm{CH}$ : (peri)centromeric, interstitial, and telomeric. With the $\mathrm{RE}+\mathrm{C}$-banding treatment, these major C-positive heterochromatin blocks could be discriminated in at least $21 \mathrm{C}$-positive heterochromatin subclasses (figure 1; and evaluation of the RE action in the major C-positive heterochromatin bands, data not shown), which are indicative of a different $\mathrm{C}$-positive heterochromatin composition reflected by a different $\mathrm{RE}$ action on the respective chromosome bands. Clearly, the $\mathrm{RE}+\mathrm{C}$ treatments are different when comparing centromeric, interstitial or telomeric bands. Furthermore, inside each major Cpositive heterochromatin class, for instance centromeric, it is possible to discriminate at least 10 autosome centromeric subclasses.

\section{Constitutive heterochromatin (C-positive heterochromatin) characterization in P. tajacu}

In figure 2, we present the C-positive heterochromatin (C- bands) for the P. tajacu chromosomes. On the majority of the autosomal chromosomes, it is possible to observe cen- tromeric C-bands. However, that is not the case on chro- mosomes 5 and 11. Autosomes 1, 2, 3, 4, 6, 7, 8, 10 and 12 reveal interstitial C-bands, and chromosomes 1 , 2, 5, 6, 7 and 8 show telomeric C-bands. The submetacentric chro- mosome 2 is the autosome that presents the highest number of C-positive heterochromatin bands. The sex chromosomes also show C-positive heterochromatin blocks. The $\mathrm{X}$ chro- mosome presents conspicuous centromeric and interstitial C- bands, but on the $\mathrm{Y}$ chromosome only a telomeric C-band is detected.

The $\mathrm{RE}+\mathrm{C}$-banding on $T$. pecari chromosomes revealed $\mathrm{CH}$ heterogeneity, and this was also observed with this approach for $P$. tajacu C-positive heterochromatin 
The arrowheads in figure 2 indicate interstitial and telom- eric C-bands revealed only by previous RE treatment. In- side each major $\mathrm{CH}$ class-(peri)centromeric, interstitial and telomeric-for each different morphological chromosome type, it was possible to discriminate at least 32 C-positive heterochromatin subclasses (figure 2; and evaluation of RE action in the major C-positive heterochromatin bands, data not shown), suggesting that this species also presents a kary- otype highly rearranged in respect of its $\mathrm{CH}$.

Interspecies constitutive heterochromatin (C-positive heterochromatin) relationship

The comparative chromosome painting results between the domestic pig and white-lipped and collared peccaries clearly revealed the high level of reshuffling between these two karyotypes (Bosma et al. 2004; Adega et al. 2006). Based on these two works, and on the assumption that the pres- ence of $\mathrm{CH}$ facilitates the occurrence of chromosomal rearrangements (Yunis and Yasmineh 1971; Peacock et al. 1982; John 1988; Chaves et al. 2004), we made a com- parative analysis between $T$. pecari and $P$. tajacu $\mathrm{CH}$ lo- calization and the breakpoints in each chromosome (figure 3). In figure 3, the corresponding breakpoints (assigned by the pig paint probes) in $T$. pecari and $P$. tajacu were named with letters in the G-banded karyotype. It was pos- sible to assign 22 breakpoints (letters A to V). The location of the $\mathrm{CH}$ bands is indicated to the right of each chromo- some. In $P$. tajacu, it was possible to correlate $\mathrm{CH}$ with 18 breakpoints. In $T$. pecari it was not possible to determine the exact correspondence for a few equivalent breakpoints (e.g. T. pecari chromosome 2 , breakpoint $\mathrm{F} / \mathrm{G}$ ). Therefore, in $T$. pecari, only four of the 20 breakpoints did not present $\mathrm{CH}$. These results suggest that $\mathrm{CH}$ could somehow be involved in the chromosome rearrangements that have occurred during the evolution of these species. Moreover, some of the breakpoints where it was not possible to assign $\mathrm{CH}$ are the same in the two species ( $P$. tajacu chromosome 1 and $T$. pecari chromosome 3 , breakpoint C; $P$. tajacu chromosome 5 and $T$. pecari chromosome 5, breakpoint N), suggestive of a further ancient rearrangement, considering the hypothesis of $\mathrm{CH}$ elimination during the process of chromosomal rearrangement.

The general comparison of the distribution and characterization of C-positive heterochromatin in the two tayassuid species suggests that the karyotypes of these species are extremely divergent. This can be seen in the present work by analysing the different actions of the same RE in the two karyotypes: e.g. AluI+C treatment generates conspicuous C-positive heterochromatin blocks in T. pecari, in contrast to the near nonexistence of C-positive heterochromatin blocks on P. tajacu chromosomes. Another important feature is that the $\mathrm{CH}$ variation seems more pronounced for $P$. tajacu than T. pecari karyotype, suggesting not only differences in the na- ture of $\mathrm{CH}$ in the two karyotypes, but also considerable het- erogeneity within each karyotype. Evidence for this comes from the higher number of $\mathrm{C}$ positive heterochromatin sub- classes identified for $P$. tajacu (32), compared to T. pecari (21), and the large number of C-positive heterochromatin subclasses detected in both species.

According to the fossil record, stratigraphy, and nu- clear and mitochondrial DNA-based evolutionary analyses (Gongora and Moran 2005), the divergence of collared and white-lipped peccaries could have taken place before the emergence and the completion of the land bridge at the Isth- mus of Panama that occurred some time in the late Pliocene, 2.5 to 3 million years ago. Thus, our data suggest that the radiation of the two species was extremely fast, and the great dissimilarity between the species is also in agreement with the results from the comparative chromosome data with pig probes (Bosma et al. 2004; Adega et al. 2006). The higher number of Cpositive heterochromatin classes/subclasses on $P$. tajacu autosomes suggests that this karyotype is more primitive, that is it did not go through a high level of homogenization due to concerted evolution of chromosomes. This karyotype resembles the ancestral form, displaying a high diversity of $\mathrm{CH}$, which is thought to facilitate the occurrence of chromosomal rearrangements (Yunis and Yasmineh 1971; Peacock et al. 1982; John 1988; Chaves et al. 2004).

Finally, the analysis of the $\mathrm{X}$ chromosome C-positive het- erochromatin in both species demonstrated that the acrocen- tric form of $T$. pecari contains a higher amount and number of C-positive heterochromatin blocks, in comparison with the submetacentric $P$. tajacu $\mathrm{X}$ chromosome; as well as a differ- ent $\mathrm{CH}$ molecular nature. In the $T$. pecari acrocentric $\mathrm{X}$ chro- mosome it was possible to identify three major C-positive heterochromatin bands, and the $M b o \mathrm{I}+\mathrm{C}$ treatment revealed an extra block at the pericentromeric region of the $\mathrm{X}$ chromo- some. The $P$. tajacu submetacentric $\mathrm{X}$ chromosome shows only two major C-positive heterochromatin blocks. These re- sults suggest that the acrocentric $\mathrm{X}$ chromosome form from $T$. pecari is the more primitive, assuming again that the presence of higher $\mathrm{CH}$ diversity is an ancestral condition resem- bling the ancestral karyotype form, not having undergone the characteristic heterochromatin homogenization of concerted evolution (Elder and Turner 1995).

\section{General remarks and conclusions}

Although the constitutive heterochromatin of the tayassuid species $P$. tajacu and $T$. pecari have been previously described by classical C-banding procedures (Pirtle 1967 
Benirschke and Kumamoto 1989), the characterization of $\mathrm{CH}$ heterogeneity is documented here for the first time for both species.

The present work demonstrated that in situ RE digestion with sequential C-banding (figures 1 and 2) could be an alternative tool for the study of $\mathrm{CH}$ heterogeneity in tayassuid chromosomes, especially when other techniques, such as fluorescence in situ hybridization with repetitive sequences (from the species in analysis), are not available.

The C-positive heterochromatin characterization of $P$. $t a-$ jacu and T. pecari autosomes suggests that the $P$. tajacu autosomal karyotype is more primitive, and has accumulated great diversity in its $\mathrm{CH}$. This idea is also supported by Gongora and Moran (2005), who performed nuclear and mitochondrial evolutionary analyses in collared, white-lipped and chacoan peccaries. These authors even suggest that the collared peccary should be classified in a separate genus within the family Tayassuidae, which is also consistent with previous analyses of cytochrome $b$ by Theimer and Keim (1998). The chromosome painting studies (Bosma et al. 2004; Adega et al. 2006) also reported an extensive karyotypic variation among the living Tayassuidae, which is in accordance with the great variation found in the $\mathrm{C}$ positive heterochromatin of $P$. tajacu and $T$. pecari karyotypes in the present study. Finally, the tayassuid X chromosome primi- tive form seems to be the one of $T$. pecari. This finding is not surprising, since the evolution of sex chromosomes is of- ten independent of that of the autosomal complement (Ohno 1967; Graves and Shetty 2000). Further studies regarding $\mathrm{CH}$ characterization, chromosome painting and other molec- ular analyses in tayassuid species will certainly contribute to a better understanding of the evolutionary processes and phylogenetic relationships within this family.

\section{Acknowledgements}

We sincerely express our gratitude to Anneke Bosma for kindly providing the fixed cell cultures of $T$. pecari used in this work. We thank Lisa Bates from Tucson Wildlife Center, Inc., and Donald DeYoung from The University of Arizona, for their kind supply of $P$. tajacu samples. This work was supported by a PhD grant (SFRH/BD/3280/2000) of the Science and Technol-ogy Foundation (FCT) of Portugal, and INVOTAN and FCT (SFRH/BSAB/329/2003) sabbatical grants.

\section{References}

Adega F., Chaves R. and Guedes-Pinto H. 2005 Chromosome restriction enzyme digestion in domestic pig (Sus scrofa). Constitutive heterochromatin arrangement. Genes Genet. Sys. 80, 4956.

Adega F., Chaves R., Kofler A., Krausman P. R., Masabanda J., Wienberg J. and Guedes-Pinto H. 2006 High resolution comparative chromosome painting in the Collared peccary (Pecari ta- jacu, Tayassuidae): a comparison with the karyotype of pig and sheep. Chromosome Res. 14, 243-251.
Benirschke K. and Kumamoto A. T. 1989 Further studies on the chromosomes of three species of peccary. Adv. Neotr. Mammal. 1, 309-316.

Bosma A. A., de Haan N. A., Arkesteijn G. J. A., Yang F., Yerle M. and Ziljstra C. 2004 Comparative chromosome painting be- tween the domestic pig (Sus scrofa) and two species of peccary, the collared peccary (Tayassu tajacu) and the whitelipped pec- cary ( $T$. pecari): a phylogenetic perspective. Cytogenet. Genome Res. 105, 115-121.

Chaves R., Guedes-Pinto H., Heslop-Harrison J. S. and Schwarzacher T. 2000 The species and chromosomal distribution of the centromeric $\alpha$-satellite I sequence from sheep in the tribe Caprini and other Bovidae. Cytogenet. Cell Genet. 91, 62-66.

Chaves R., Adega F., Santos S., Heslop-Harrison J. S. and Guedes- Pinto H. 2002 In situ hybridization and chromosome banding in mammalian species. Cytogenet. Genome Res. 96, 113-116.

Chaves R., Santos S. and Guedes-Pinto H. 2004 Comparative anal- ysis (Hippotragini versus Caprini, Bovidae) of Xchromosome's constitutive heterochromatin by in situ restriction endonuclease digestion: X-chromosome constitutive heterochromatin evolu- tion. Genetica 121, 315325.

Dimitri P., Corradini P., Rossi F. and Verni F. 2004 The paradox of functional heterochromatin. Bioessays 27, 29-41.

Elder J. F. Jr and Turner B. J. 1995 Concerted evolution of repetitive DNA sequences in eukaryotes. Q. Rev. Biol. 70, 297320.

Giannoni M. A., Giannoni M. L. and Ferrari I. 1982a Kary- otype evolution in the Suidae family. I. Chromosomal similar- ities among Sus and Tayassu species. In. Proceedings of the Fifth European Colloquium on Cytogenetics of Domestic Ani- mals. Ricerca scientifica ed educazione permanente (Università degli Studi di Milano), pp. 288-297.

Giannoni M. A., Giannoni M. L. and Ferrari I. 1982b Karyotype evolution in the Suidae family. II. Chromosomal similarities among Tayassu species. In Proceedings of the Fifth European Colloquium on Cytogenetics of Domestic Animals. Ricerca sci- entifica ed educazione permanente (Università degli Studi di Mi-lano), pp. 298-311.

Gongora J. and Moran C. 2005 Nuclear and mitochondrial evolutionary analyses of Collared, White-lipped, and Chacoan pecca- ries (Tayassuidae). Mol. Phylogenet. Evol. 34, 181-189.

Gosálvez J., Mezzanotte R., López-Fernández C., De Castillo P., Stockert J. C., Goyanes V. and Sumner A. T. 1991 Selective di- gestion of mouse chromosomes with restriction endonucleases. II. X-ray microanalysis of HaeIII-treated chromosomes. Cyto- genet. Cell Genet. 56, 82-86.

Gosálvez J., López-Fernández C., Goyanes V. and Mezzanotte R. 1997 Chromosome differentiation using nucleases: an overview. Chromosomes Today, 12, 23-49.

Graves J. A. M. and Shetty S. 2000 The evolution of sex chromosomes in higher vertebrates. In Comparative genomics (ed. M. Clark), pp. 153-205. Kluwer, Boston.

Hufty M. P., Sedgwick C. J. and Benirschke K. 1973 The karyotypes of the white-lipped and collared peccaries. Aspects of their chro- mosomal evolution. Genen Phaenen 16, 81-86.

John B. 1988 The biology of heterochromatin. In Heterochromatin: molecular and structural aspects (ed. R. S. Verma), pp. 1-147. Cambridge University Press, New York. 
Nieddu M., Rossino R., Pichiri G., Rocchi M., Setzu M. D. and Mezzanotte R. 1999 The efficiency of in situ hybridization on human chromosomes with alphoid DNAs is enhanced by previous digestion with AluI and TaqI. Chromosome Res. 7, 593-602.

Ohno S. 1967 Sex chromosomes and sex linked genes. Springer, Berlin.

Peacock W. J., Dennis E. S. and Gerlach W. L. 1982 DNA se- quence changes and speciation. In Mechanisms of speciation (ed. C. Barigozzi), pp. 123-142. Alan R. Liss, New York.

Pieczarka J. C., Nagamachi C. Y., Muniz J. A. P. C., Barros R. M. S. and Mattevi M. S. 1998 Analysis of constitutive heterochromatin of Aotus (Cebidae, Primates) by restriction enzyme and fluorochrome bands. Chromosome Res. 6, 77-83.

Pirtle E. C. 1967 Chromosomes of the female peccary (Tayassu tajacu). Mamm. Chromosome Newslett. 8, 16.

Rocco L., Morescalchi M. A., Costagliola D. and Stingo V. 2002 Karyotype and genome characterization in four cartilaginous fishes. Gene 295, 289-298.

Schmid M., Haaf T., Steinlein C., Nanda I. and Mahony M. 2002 Chromosome banding in Amphibia. XXV. Karyotype evolution and heterochromatin characterization in Australian Mixophyes (Anura, Myobatrachidae). Cytogenet. Genome Res. 97, 239-253.

Sumner A. T. 1972 A simple technique for demonstrating centromeric heterochromatin. Exp. Cell Res. 75, 304-306.

Theimer T. C. and Keim K. 1998 Phylogenetic relationships of peccaries based on mitochondrial cytochrome b DNA sequences. $J$. Mammal. 79, 566-572.

Verma R. S. and Babu A. 1995 Human chromosomes: principles and techniques. McGraw-Hill, New York.

Yunis J. J. and Yasmineh W. G. 1971 Heterochromatin, satellite DNA and cell function. Science 174, 1200-1209.

Zijlstra C., Mellink C. H. M., De Haan N. A. and Bosma A. A. 1997 Localization of the 18S, 5.8S and 28S rRNA genes and the 5S rRNA genes in the babirusa and the white-lipped peccary. $C y$ togenet. Cell Genet. 77, 273-277. 


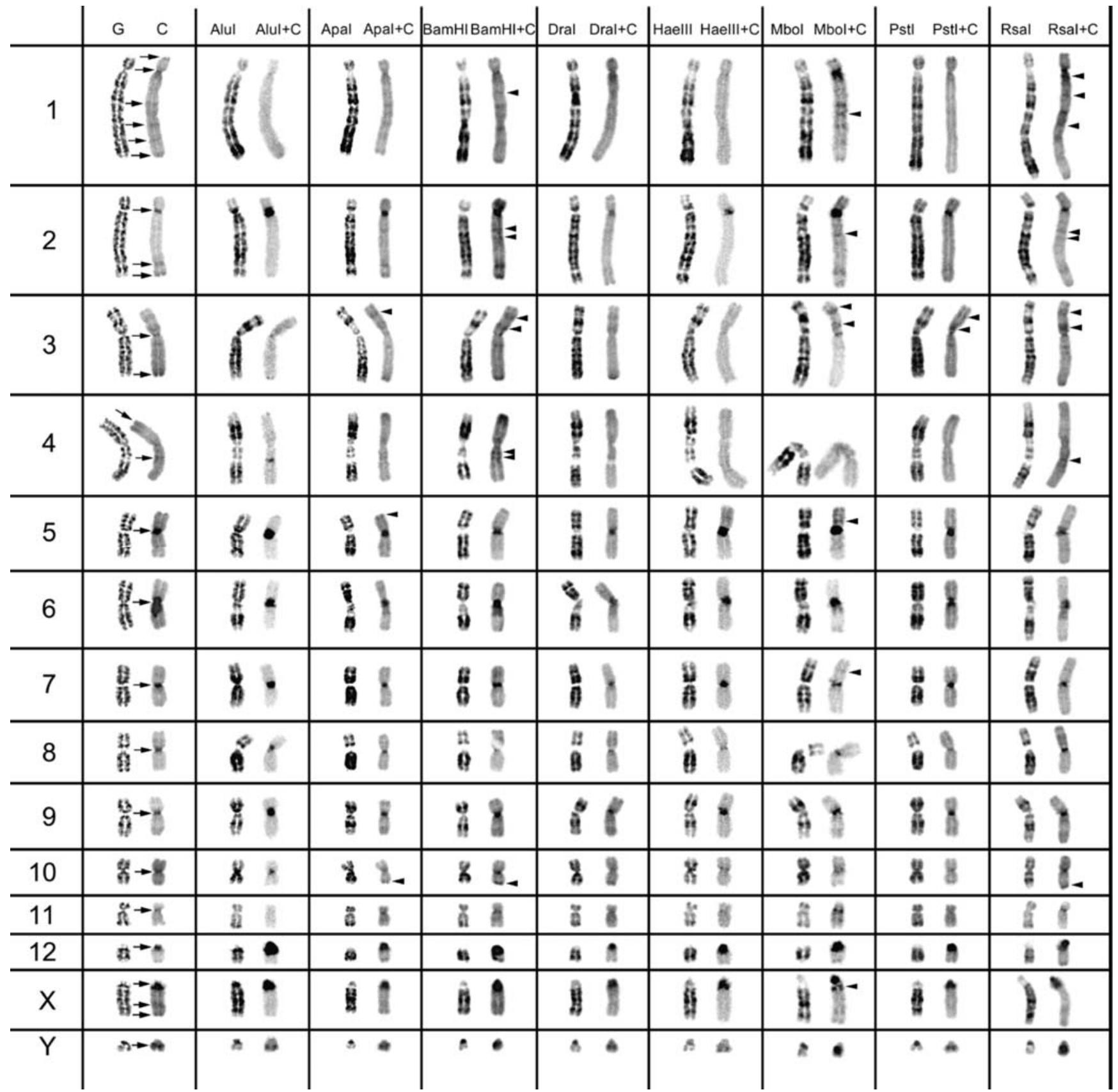

Figure 1. Control G-banding and C-banding of Tayassu pecari chromosomes are shown on the left, with the classical C-bands indicated by arrows. The other columns show the bands produced by the eight different restriction endonucleases, before and after Cbanding (arrowheads indicate C-positive heterochromatin bands only revealed by previous RE treatment). 


\begin{tabular}{|c|c|c|c|c|c|c|c|c|c|}
\hline & G C & Alul Alul+C & Apal Apal+C & BamHI BamHI+C & Dral Dral+C & HaellI HaellI+C & Mbol Mbol+C & Pstl $\quad P_{s t t+C}$ & Rsal Rsal+C \\
\hline 1 & & $\hat{\theta}^{2}$ & i & & & & & है & ${ }^{3}{ }^{3}$ \\
\hline 2 & & 盟量 & : & 출 & 룰 & & $\frac{5}{6}$ & है & $\begin{array}{l}8 \\
8 \\
8\end{array}$ \\
\hline 3 & 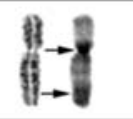 & है & : & a & 68 & $\vec{a}$ & कै & है & \#ै \\
\hline 4 & $\stackrel{y}{y=0}$ & 娄 & ? & $\frac{5}{4}$ & है & 章 & है & 8 & 8 \\
\hline 5 & 8 & 急 & 8 is & 负娄 & $<2$ & 88 & मै & 6. & 6. \\
\hline 6 & $\begin{array}{l}y=0 \\
y \rightarrow 0\end{array}$ & है & 8 & 骨 है & 6 & $n$ & ะ & 8 & 8 \\
\hline 7 & 한? & 8 & है & 落 & है & 뾸 & 过 & 를 & 一类 \\
\hline 8 & $A^{\infty}=\mathbb{R}^{\prime}$ & 83 & 8 & 챌 & \& 8 & ถ้ & 8 & ํำ & 8 \\
\hline 9 & $\ddot{B} \rightarrow \vec{B}$ & $i a$ & 38 & 68 & क 5 & 8 क & $=8$ & की & $\therefore$ है \\
\hline 10 & $\ddot{B} \rightarrow$ & $\Rightarrow 2$ & $=$ & $\Rightarrow \mathbf{s}$ & $=3$ & $6 \pi$ & $\approx$ 패. & \& 8. & is \\
\hline 11 & है है & 6 5 & 58 & a & $A$ ह & ค 8 & o t 할 & 5 & \\
\hline 12 & 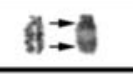 & 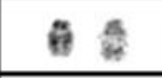 & 68 & कै & 6 츨 & 해 & $\therefore 8$ & if & a \\
\hline 13 & $\stackrel{5}{\circ \rightarrow 8}$ & i $\frac{8}{2}$ & A 8 & \& 6 & $\approx A$ & A $\mathrm{x}$ & 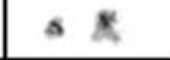 & A S & a 8 \\
\hline 14 & $a \rightarrow \infty$ & $\Delta \leqslant$ & $\therefore=$ & $\rightarrow B$ & $4 \mathrm{a}$ & 56 & 48 & -8 & a \\
\hline$x$ & 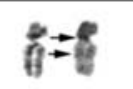 & $8 \mathbb{E}^{-}$ & 8 & 量 & है & 58 & है & \& & 常 \\
\hline$Y$ & $b_{h \rightarrow C}$ & 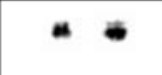 & A C & $6 a$ & $\cdot \Delta$ & $=8$ & .6 & 60 & \\
\hline
\end{tabular}

Figure 2. Control G-banding and C-banding of Pecari tajacu chromosomes are shown on the left, with the classical C-bands indicated by arrows. The other columns show the bands produced by the eight different restriction endonucleases, before and after $\mathrm{C}$ banding (arrowheads indicate C-positive heterochromatin bands only revealed by previous RE treatment). 


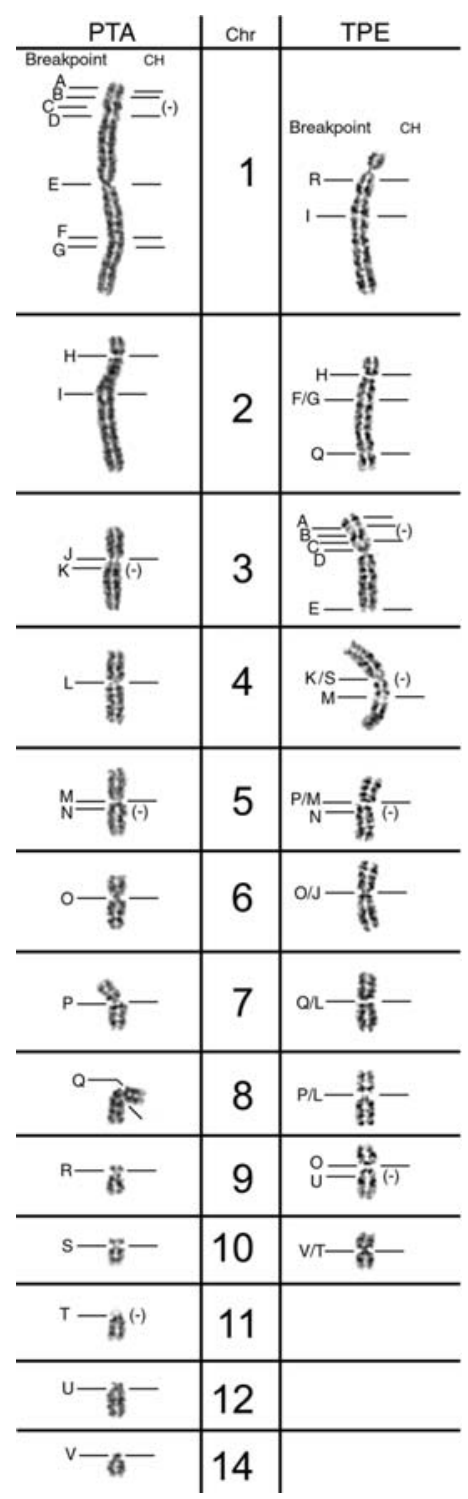

Figure 3. Homologous breakpoints in Pecari tajacu (PTA) and Tayassu pecari (TPE) (assigned by comparative chromosome painting with pig probes) named with letters (A to $\mathrm{V}$ ) in the G-banded karyotypes. The location of the $\mathrm{CH}$ bands is indicated to the right of each chromosome by a dash. 